\title{
Mean platelet volume provides beneficial diagnostic and prognostic information for patients with resectable gastric cancer
}

\author{
XIAO-MING SHEN ${ }^{1 *}$, YOU-YOU XIA ${ }^{2,3^{*}}$, LIAN LIAN $^{1,4^{*}}$, CHONG ZHOU $^{5}$, XIANG-LI LI ${ }^{6}$, SHU-GUANG HAN $^{6}$, \\ YAN ZHENG ${ }^{1}$, FEI-RAN GONG ${ }^{7}$, MIN TAO $^{4,8-10}$, ZHONG-QI MAO ${ }^{11}$ and WEI LI ${ }^{4,8,10}$ \\ ${ }^{1}$ Department of Oncology, Suzhou Xiangcheng People's Hospital, Suzhou; ${ }^{2}$ Department of Radiation Oncology, \\ Lianyungang First People's Hospital, Lianyungang; ${ }^{3}$ The Fourth Clinical Medical College, Nanjing Medical University, \\ Nanjing; ${ }^{4}$ Department of Oncology, The First Affiliated Hospital of Soochow University, Suzhou; ${ }^{5}$ Department of \\ Radiation Oncology, Xuzhou Central Hospital, Xuzhou; ${ }^{6}$ Department of General Surgery, Suzhou Xiangcheng \\ People's Hospital; ${ }^{7}$ Department of Hematology, The First Affiliated Hospital of Soochow University; ${ }^{8}$ Jiangsu Institute \\ of Clinical Immunology; ${ }^{9}$ Institute of Medical Biotechnology, Soochow University; ${ }^{10}$ PREMED Key Laboratory \\ for Precision Medicine, Soochow University; ${ }^{11}$ Department of Minimally Invasive Surgery, \\ The First Affiliated Hospital of Soochow University, Suzhou, Jiangsu, P.R. China
}

Received January 5, 2016; Accepted July 26, 2016

DOI: $10.3892 / \mathrm{ol} .2016 .4913$

\begin{abstract}
Gastric cancer is the fourth most frequent cancer and the second cause of cancer-related mortalities worldwide. Platelets play an important and multifaceted role in cancer progression. Elevated mean platelet volume (MPV) detected in peripheral blood has been identified in various types of cancer. In the present study, we investigated the application value of MPV in early diagnostic and prognostic prediction in patients with resectable gastric cancer. In total, 168 patients with resectable gastric cancer were included and separated into the gastric cancer and healthy control groups according to median preoperatic MPV value (MPV low, $<10.51$ or MPV high, $\geq 10.51$ ). The results showed that the pre-operatic MPV level was significantly higher in gastric cancer patients compared with the healthy subjects. Low pre-operatic MPV level correlated with improved clinicopathological features, including decreased depth of invasion, less lymphonodus metastasis and
\end{abstract}

Correspondence to: Dr Wei Li, Department of Oncology, The First Affiliated Hospital of Soochow University, 188 Shizi Street, Suzhou, Jiangsu 215006, P.R. China

E-mail: liwei10@suda.edu.cn

Dr Zhong-Qi Mao, Department of Minimally Invasive Surgery, The First Affiliated Hospital of Soochow University, 188 Shizi Street, Suzhou, Jiangsu 215006, P.R. China

E-mail: maozq31@hotmail.com

*Contributed equally

Key words: mean platelet volume, gastric cancer, diagnosis, prognosis early tumor stage. The Kaplan-Meier plots showed that the patients with higher pre-operatic MPV had decreased overall survival (OS) and disease-free survival (DFS). Surgical tumor resection resulted in a significant decrease in the MPV level. The patients whose MPV level decreased following surgery had an improved OS. Multivariate Cox regression analysis revealed that the depth of invasion, lymphonodus metastasis, American Joint Committee on Cancer (AJCC) stage, and changes in MPV following surgery were prognostic factors affecting OS, and the AJCC stage and pre-operatic MPV were prognostic factors affecting DFS. In conclusion, MPV measurement can provide important diagnostic and prognostic results in patients with resectable gastric cancer.

\section{Introduction}

Gastric cancer is the fourth most common cancer and the second most common cause of cancer-related mortalities (1). Delayed diagnosis is the principal cause of increased mortality and morbidity associated with this type of cancer. At the time of diagnosis, only $25 \%$ of patients are able to undergo surgical resection. The 5-year survival rate is only $10-15 \%$ in individuals with advanced disease (1). Therefore, early diagnosis is crucial, especially given that early symptoms (dyspepsia, mild epigastric pain, nausea, and anorexia) are not very specific. Biomarkers including, CEA and CA 19-9, have been tested. However, these biomarkers have a low diagnostic ability to detect gastric cancer (2). Therefore, identification of novel biomarkers for the diagnosis and follow-up of gastric cancer is essential.

Platelets play an important and multifaceted role in cancer progression (3). Previous findings suggested that platelets accelerate the natural course of cancer by promoting neoangiogenesis, degradation of the extracellular matrix, 
Table I. Relationship between pre-operative MPV and demographic and clinical parameters.

\begin{tabular}{|c|c|c|c|c|c|}
\hline Parameters & No. of patients & $\begin{array}{c}\text { Low MPV }(<10.51), \\
\text { no. of patients }\end{array}$ & $\begin{array}{c}\text { High MPV }(\geq 10.51), \\
\text { no. of patients }\end{array}$ & $\chi^{2}$ & P-value \\
\hline \multicolumn{6}{|l|}{ Gender } \\
\hline Male & 116 & 62 & 54 & \multirow[t]{2}{*}{1.7825} & \multirow[t]{2}{*}{0.1818} \\
\hline Female & 52 & 22 & 30 & & \\
\hline \multicolumn{6}{|l|}{ Age (years) } \\
\hline$<65$ & 96 & 45 & 51 & \multirow[t]{2}{*}{0.8750} & \multirow[t]{2}{*}{0.3496} \\
\hline$\geq 65$ & 72 & 39 & 33 & & \\
\hline \multicolumn{6}{|l|}{ Tumor size (cm) } \\
\hline$<5$ & 108 & 51 & 57 & \multirow[t]{2}{*}{0.9333} & \multirow[t]{2}{*}{0.3340} \\
\hline$\geq 5$ & 60 & 33 & 27 & & \\
\hline \multicolumn{6}{|l|}{ Lauren type } \\
\hline Intestinal & 97 & 50 & 47 & \multirow[t]{2}{*}{0.2195} & \multirow[t]{2}{*}{0.6394} \\
\hline Diffuse & 71 & 34 & 37 & & \\
\hline \multicolumn{6}{|l|}{ Depth of invasion } \\
\hline $\mathrm{T} 1, \mathrm{~T} 2$ & 66 & 15 & 51 & \multirow[t]{2}{*}{32.3422} & \multirow[t]{2}{*}{$<0.001^{\mathrm{a}}$} \\
\hline $\mathrm{T} 3, \mathrm{~T} 4$ & 102 & 69 & 33 & & \\
\hline \multicolumn{6}{|l|}{ Lymph node metastases } \\
\hline N0, N1 & 54 & 12 & 42 & \multirow[t]{2}{*}{24.5614} & \multirow[t]{2}{*}{$<0.001^{\mathrm{a}}$} \\
\hline $\mathrm{N} 2, \mathrm{~N} 3$ & 114 & 72 & 42 & & \\
\hline \multicolumn{6}{|l|}{ Degree of differentiation } \\
\hline Highly differentiated & 50 & 23 & 27 & \multirow[t]{2}{*}{0.4556} & \multirow[t]{2}{*}{0.4997} \\
\hline $\begin{array}{l}\text { Moderately or poorly } \\
\text { differentiated }\end{array}$ & 118 & 61 & 57 & & \\
\hline \multicolumn{6}{|l|}{ AJCC stage } \\
\hline I, II & 57 & 12 & 45 & \multirow[t]{2}{*}{28.9161} & \multirow[t]{2}{*}{$<0.001^{\mathrm{a}}$} \\
\hline III, IV & 111 & 72 & 39 & & \\
\hline
\end{tabular}

${ }^{\mathrm{a}} \mathrm{P}<0.05$, statistically significant. MPV, mean platelet volume; AJCC, American Joint Committee on Cancer.

release of adhesion molecules, and growth factors, thus providing essential components for tumor growth and metastatic spread (4). The presence of platelets is increased by proinflammatory cytokines released by cancer cells through the promotion of megakaryocyte proliferation (5). Given the relationship between platelet and cancer, platelet-based markers are potential candidates for the diagnosis and follow-up of gastric cancer. Elevated mean platelet volume (MPV) of peripheral blood has been identified in various types of cancer, including hepatocellular carcinoma (6), ovarian (7), colon (8) lung and breast (9) cancer. In the present study, we examined whether MPV is suitable as a diagnostic and prognostic marker for the detection of resectable gastric cancer.

\section{Materials and methods}

Patients. The study was conducted as a retrospective study of patients with gastric cancer who had been referred to the First Affiliated Hospital of Soochow University between January, 2007 and January, 2010. Approval for the study was granted by the Medical Ethics Committees of the First Affiliated Hospital of Soochow University (Jiangsu,
China). Patients with hypertension, hematological and renal disease, heart failure, chronic infection, hepatic disorder and other cancer types were excluded from the study. In total, 168 patients with resectable gastric cancer were recruited in this study. Patient characteristics are presented in Table I. The mean age (range) of study patients was 56.5 (31-82) years. The staging of cancer was determined according to the tumornode-metastasis (TNM) classification, using the American Joint Committee on Cancer (AJCC) recommendations (10). The patients were followed regularly for 60 months. Thirty age- and gender-matched healthy individuals were also included in the present study.

Blood analysis. Peripheral venous blood (5-7 ml) was collected into sterile EDTA tubes. Blood specimens were obtained in the morning between 06:30 and 07:30 a.m. to minimize the impact of circulating hormones (circadian rhythm) on the number and subtype distribution of white blood cells. Haematological parameters were analyzed within $30 \mathrm{~min}$ after blood collection using a haematology analyser Sysmex XE-2100 (Sysmex, Kobe, Japan). MPV was thus obtained and used in subsequent analyses. 
Heathy subjects Gastric cancer patients

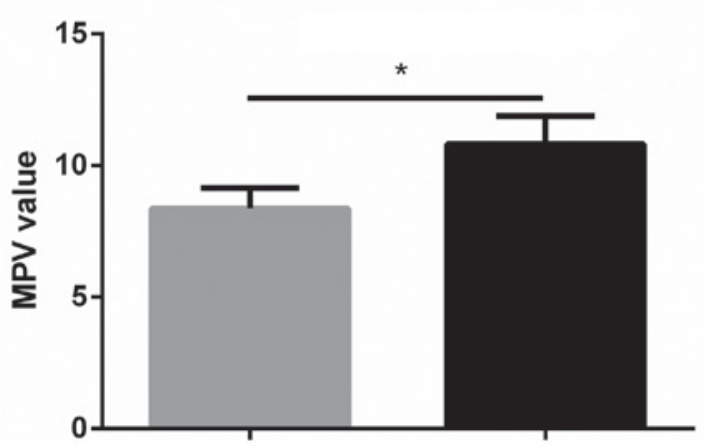

Figure 1. Comparison of pre-operative MPV values between healthy controls and patients with gastric cancer. Measurement data are expressed by mean \pm standard. ${ }^{*} \mathrm{P}<0.001$ vs. healthy controls. MPV, mean platelet volume.

Statistical analysis. Statistical analyses were performed using SPSS 19.0 software (SPSS, Inc., Chicago, IL, USA). Measurement data were presented as mean \pm standard variation. The association between MPV and clinicopathological features were tested using the Chi-square test. For the analysis of survival data, Kaplan-Meier curves were constructed, and statistical analysis was carried out using the log-rank test. The prognostic analyses were performed as disease-free survival (DFS) and overall survival (OS). OS was defined as the time from the diagnosed date to death from any cause. DFS was defined as the time from the primary operation to the relapse of tumor. The multivariate Cox regression was performed for each outcome parameter, using a backwards elimination technique to derive a potentially suitable set of predictors. $\mathrm{P}<0.05$ was considered to indicate statistically significant results.

\section{Results}

Pre-operative MPV is higher in patients with gastric cancer patients compared with healthy controls. The mean pre-operative MPV in the study patients was $10.82 \pm 1.06$, which was significantly higher than that in the healthy individuals (8.37 $\pm 0.78, p<0.001$; Fig. 1). This result indicated that MPV is useful in the early diagnosis of gastric cancer.

Low pre-operative MPV level predicts better outcomes. As shown in Table I, pre-operative MPV levels inversely correlated with clinicopathological parameters, including depth of invasion, lymphonodus metastasis and the AJCC stage.

Median OS for all the patients was 57 months, whereas the median was DFS 27 months (Fig. 2A).

The patients were separated into two groups according to median pre-operative MPV: low $(<10.51)$ and high $(\geq 10.51)$ MPV. The Kaplan-Meier plots showed the association between pre-operative MPV and OS and PFS (Fig. 2B and C). The OS and PFS rates of high pre-operative MPV were 29.8 and $11.9 \%$, respectively, and were significantly different from corresponding rates in the low pre-operative MPV group (46.7 and $24.3 \%$, respectively, both $\mathrm{p}<0.01)$. This result demonstrated that patients with higher pre-operative MPV had decreased survival rates.
A

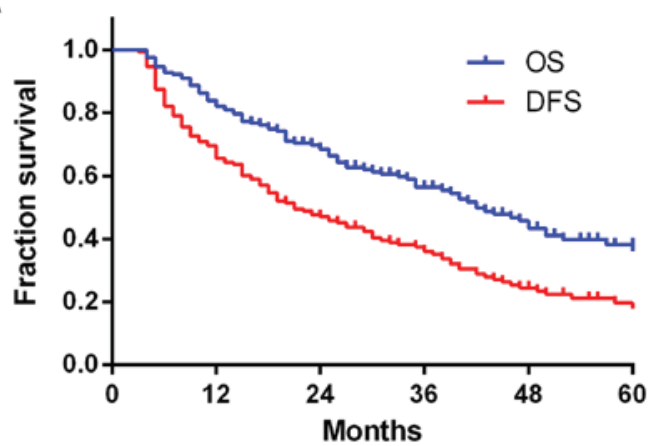

B

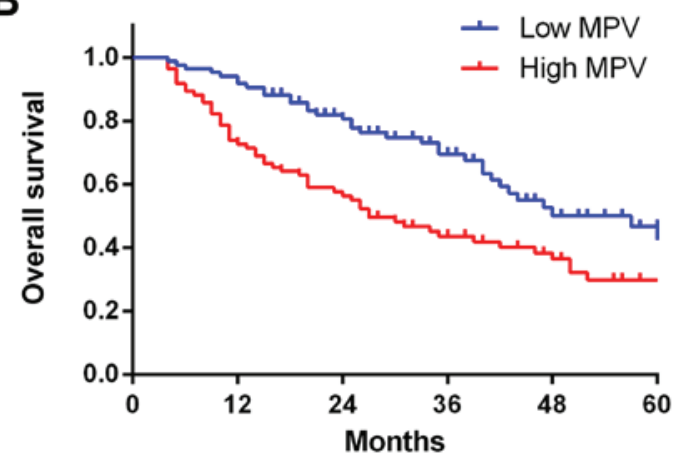

C

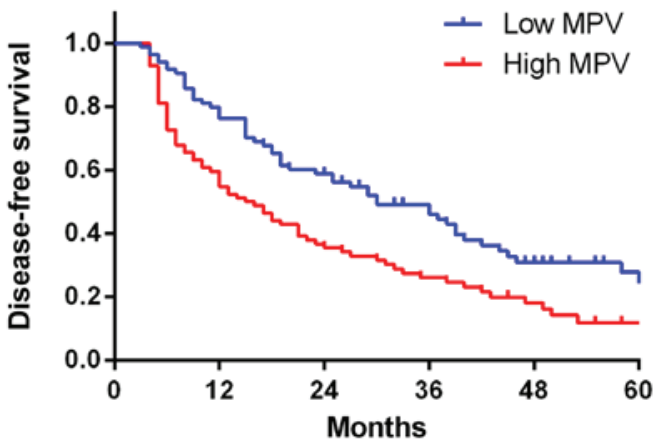

Figure 2. Association between pre-operative MPV, and OS and DFS (A) Predicted probability of OS and DFS. (B) OS stratified based on low or high pre-operative MPV. (C) DFS stratified based on low or high pre-operative MPV. MPV, mean platelet volume; OS, overall survival; DFS, disease-free survival.

MPV changes before and after surgery predict improved outcomes. MPV increased significantly 3-4 days following surgery and returned to pre-operation levels one week after the surgery (Fig. 3A). Furthermore, surgical tumor resection led to a significant decrease in the average MPV one month after surgery (Fig. 3A).

When individual MPV changes were evaluated, it was observed that a decrease was present in 99 patients and absent in the remaining 69 patients. The Kaplan-Meier plots indicating an association between MPV values and OS are shown in Fig. 3B. It was evident that OS was improved in patients whose MPV decreased after surgery, compared with those without any change (40.5 vs. $28.9 \%$, p<0.0037; Fig. 3B).

Univariate and multivariate analysis of risk factors for OS and DFS. Univariate and multivariate analyses were performed 

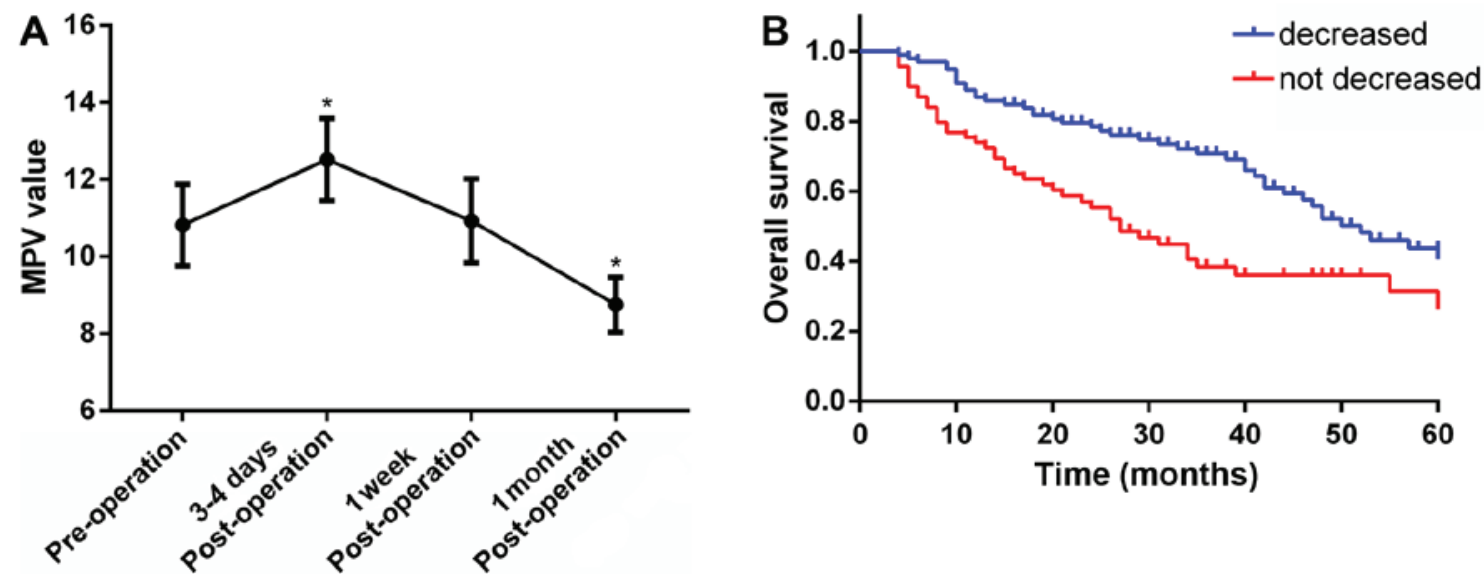

Figure 3. Association between changes in MPV values and overall survival. (A) Pre-operative vs. post-operative MPV values. Measurement data are presented as mean \pm standard. " $\mathrm{P}<0.001$ vs. pre-operation. (B) OS stratified based on post-operative decrease in MPV values. MPV, mean platelet volume; OS, overall survival; DFS, disease-free survival.

to identify the risk factors associated with OS and DFS. As shown in Table II, univariate analysis revealed that 5 of 10 risk factors affected OS and DFS. These factors included depth of invasion, lymphonodus metastasis, AJCC stage, pre-operatic MPV, and changes in MPV after surgery. Multivariate analysis further confirmed that depth of invasion, lymphonodus metastasis, AJCC stage, and changes in MPV following surgery were the factors associated with OS. Furthermore, AJCC stage and pre-operative MPV were the prognostic factors for DFS.

\section{Discussion}

The involvement of platelets and coagulation factors in hematogenous tumor metastasis are well known. Elevated thrombocytosis and platelet counts are associated with advanced, often metastatic, stages of cancer and to be negative prognostic markers for various types of cancer, including endometrial carcinoma, cervical, ovarian, gastric, and esophageal cancer (11).

Platelets participate in multiple steps of hematogenous metastasis. Covered with platelets, circulating cancer cells can transport more easily in the bloodstream and overcome countering effects of immune cells and physical factors such as shear force and mechanical trauma due to passage through microvasculature $(12,13)$. Platelets can also promote tumor growth by increasing angiogenesis via the cytokine vascular endothelial growth factor (VEGF). The platelet content of VEGF is significantly elevated in cancer patients, and there is a direct correlation between the number of circulating platelets and the level of serum VEGF (3).

The cancer-promoting effects of platelets are amplified by stimulation of activation and aggregation of platelets caused by proinflammatory cytokines released by cancer cells (4). This involves proliferation and differentiation of early progenitor cells such as megakaryocyte progenitors. In addition, malignant cells possess the ability of aggregating platelets through a process known as tumor cell-induced platelet aggregation (TCIPA) (12). Therefore, the close involvement of the platelet with metastasizing cancer makes this cell type a promising candidate for early cancer diagnosis and treatment (11).
Large platelets are more reactive than their smaller counterparts, and are more likely to aggregate, leading to thrombosis. Large platelets are an independent risk factor for myocardial infarction, as platelet size is a predictor of recurrent myocardial infarction and death (14). The MPV tested in our study can be easily evaluated by hematological analyzers, which makes it a convenient marker of platelet functions and activation (3). Elevated MPV may indicate a tendency towards thrombosis, as it has been demonstrated for myocardial infarction and cerebrovascular embolus (15). Previous studies suggested that MPV is a potential biomarker for the diagnosis and follow-up of types of cancer (6-9).

Elevated MPV values may be a consequence of systemic inflammatory response (16), which is believed to play a critical role in the development and progression of different cancers by promoting cancer cell proliferation and survival, angiogenesis, cancer metastasis and modulating cancer cell response to therapies (3). Numerous types of cancer release proinflammatory cytokines, such as interleukin (IL)-1, IL-3, and IL-6, which promote the proliferation of megakaryocytes, resulting in platelet activation and aggregation (4). In ovarian cancer, elevated levels of IL-6 in ascites and cyst fluids have been associated with thrombocytosis. Furthermore, administration of recombinant IL- 6 has been associated with increased platelet count (5). Elevated IL-6 is significantly higher in individuals with gastric cancer, as well as in patients with prostate cancer $(17,18)$.

The close interplay between inflammation, coagulation, and cancer progression ignited intensive studies in this field (4). For example, long-term use of non-steroid anti-inflammatory drugs such as aspirin is associated with a reduced risk of esophageal cancer (1). Clinical and epidemiological studies demonstrated an association between chronic inflammation and gastric cancer (19-21). Based on these considerations, we postulated that elevated MPV values in patients with gastric cancer may be a consequence of systemic inflammatory response. Correspondingly, a decrease of MPV values after surgery may be due to a decreased systemic inflammatory response. Therefore, patients whose MPV values did not decrease after surgical resection of the cancer may continue to 


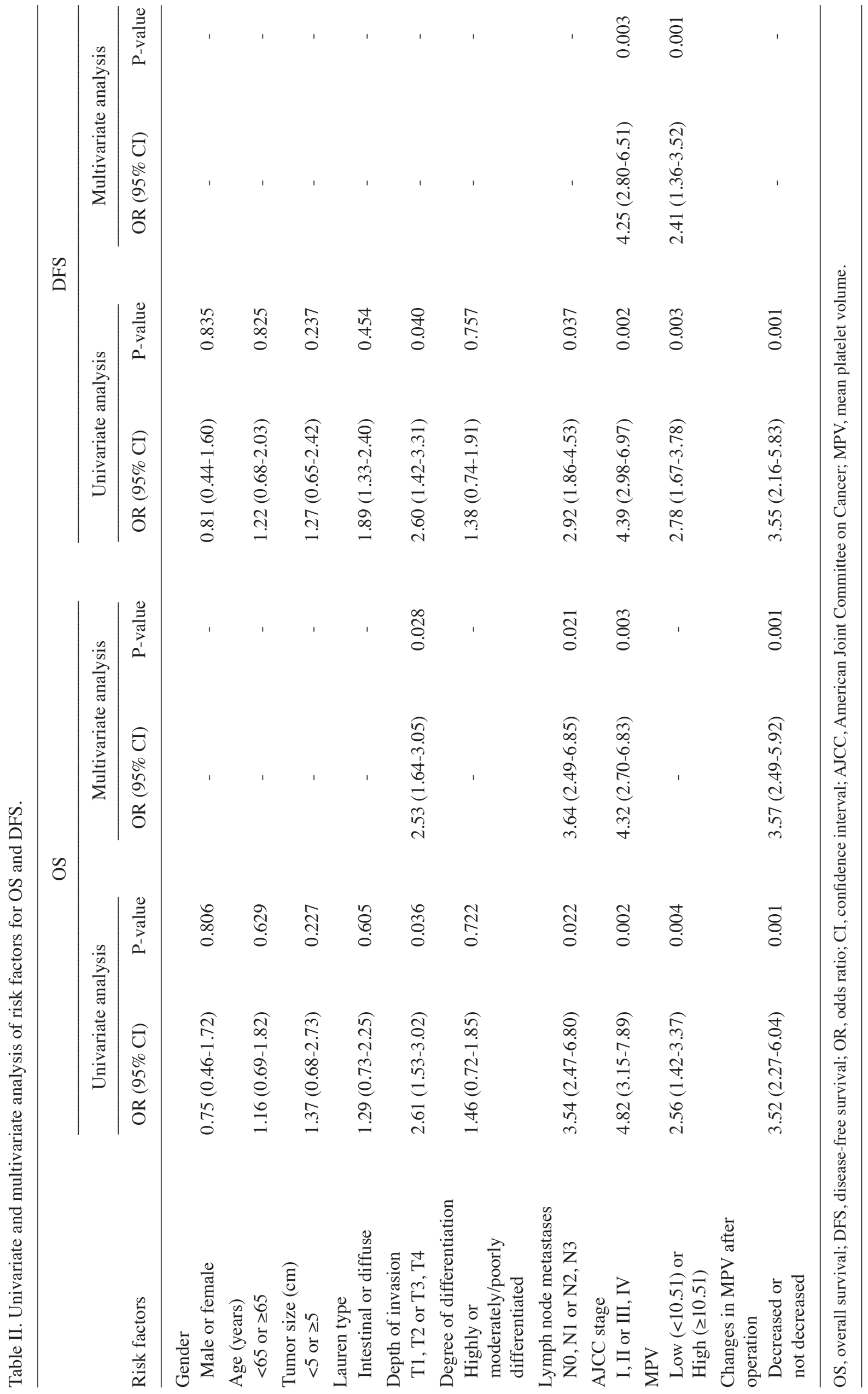


harbor untamed systemic inflammatory response, leading to an unfavourable prognosis.

In conclusion, the present study indicates an association of the pre-operative MPV level and changes between pre- and post-operative levels with the diagnosis and prognosis of gastric cancer. Although MPV is also a non-specific marker, this non-invasive, convenient and inexpensive biomarker may be a complement to the present biomarkers, and a benefit to the early detection and prognosis evaluation of gastric cancer.

\section{Acknowledgements}

The present study was supported by the National Natural Science Foundation of China (grant nos. 81472296, 81101867, 81272542,81200369 and 81372443), the China International Medical Foundation (grant no. CIMF-F-H001-057), the Special Foundation of Clinical Medicine of Jiangsu Provincial Bureau of Science and Technology (grant no. BL2014039), the Scientific Research Project of Jiangsu Provincial Bureau of Traditional Chinese Medicine (grant no. L213236), the Medical Scientific Research Project of Jiangsu Provincial Bureau of Health (grant no. Z201206), the Special Foundation of Wu Jieping Medical Foundation for Clinical Scientific Research (grant nos. 320.6753.1225 and 320.6750.12242), the Science and Education for Health Foundation of Suzhou for Youth (grant nos. SWKQ1003 and SWKQ1011), the Science and Technology Project Foundation of Suzhou (grant nos. SYS201112, SYSD2012137 and SYS201335), the Science and Technology Foundation of Suzhou Xiangcheng (grant nos. SZXC2012-70 and XJ201451), and a Project Founded by the Priority Academic Program Development of Jiangsu Higher Education Institutions.

\section{References}

1. Wang WH, Huang JQ, Zheng GF, Lam SK, Karlberg J and Wong BC: Non-steroidal anti-inflammatory drug use and the risk of gastric cancer: a systematic review and meta-analysis. J Natl Cancer Inst 95: 1784-1791, 2003.

2. Mroczko B, Groblewska M, Łukaszewicz-Zajac M, Bandurski R, Kedra B and Szmitkowski M: Pre-treatment serum and plasma levels of matrix metalloproteinase 9 (MMP-9) and tissue inhibitor of matrix metalloproteinases 1 (TIMP-1) in gastric cancer patients. Clin Chem Lab Med 47: 1133-1139, 2009.

3. Kemal Y, Yucel I, Ekiz K, Demirag G, Yilmaz B, Teker F and Ozdemir M: Elevated serum neutrophil to lymphocyte and platelet to lymphocyte ratios could be useful in lung cancer diagnosis. Asian Pac J Cancer Prev 15: 2651-2654, 2014.

4. Seretis C, Seretis F, Lagoudianakis E, Politou M, Gemenetzis G and Salemis NS: Enhancing the accuracy of platelet to lymphocyte ratio after adjustment for large platelet count: a pilot study in breast cancer patients. Int J Surg Oncol 2012: 653608, 2012. doi: $10.1155 / 2012 / 653608$.
5. Heras P, Hatzopoulos A, Kritikos N and Kritikos K: Platelet count and tumor progression in gastric cancer patients. Scand J Gastroenterol 45: 1005-1006, 2010.

6. Cho SY, Yang JJ, You E, Kim BH, Shim J, Lee HJ, Lee WI, Suh JT and Park TS: Mean platelet volume/platelet count ratio in hepatocellular carcinoma. Platelets 24: 375-377, 2013.

7. Kemal Y, Demirağ G, Ekiz K and Yücel I: Mean platelet volume could be a useful biomarker for monitoring epithelial ovarian cancer. J Obstet Gynaecol 34: 515-518, 2014.

8. Mutlu H, Berk V, Karaca H, Erden A, Aslan T and Akca Z: Treatment regimen with bevacizumab decreases mean platelet volume in patients with metastatic colon cancer. Clin Appl Thromb Hemost 18: 546-548, 2012.

9. Aksoy S, Kilickap S, Hayran M, Harputluoglu H, Koca E, Dede DS, Erman M and Turker A: Platelet size has diagnostic predictive value for bone marrow metastasis in patients with solid tumors. Int J Lab Hematol 30: 214-219, 2008.

10. Edge SB and Compton CC: The American Joint Committee on Cancer: the 7th edition of the AJCC cancer staging manual and the future of TNM. Ann Surg Oncol 17: 1471-1474, 2010.

11. Erpenbeck L and Schön MP: Deadly allies: the fatal interplay between platelets and metastasizing cancer cells. Blood 115: 3427-3436, 2010.

12. Lian L, Li W, Li ZY, Mao YX, Zhang YT, Zhao YM, Chen K, Duan WM and Tao M: Inhibition of MCF-7 breast cancer cell-induced platelet aggregation using a combination of antiplatelet drugs. Oncol Lett 5: 675-680, 2013.

13. Shou LM, Zhang QY, Li W, Xie X, Chen K, Lian L, Li ZY, Gong FR, Dai KS, Mao YX, et al: Cantharidin and norcantharidin inhibit the ability of MCF-7 cells to adhere to platelets via protein kinase $\mathrm{C}$ pathway-dependent downregulation of $\alpha 2$ integrin. Oncol Rep 30: 1059-1066, 2013.

14. Karagöz B, Bilgi O, Alacacioğlu A, Ozgün A, Sayan O, Erikçi AA and Kandemir EG: Mean platelet volume increase after tamoxifen, but not after anastrazole in adjuvant therapy of breast cancer. Med Oncol 27: 199-202, 2010.

15. Mutlu H, Artis TA, Erden A and Akca Z: Alteration in mean platelet volume and platicrit values in patients with cancer that developed thrombosis. Clin Appl Thromb Hemost 19: 331-333, 2013.

16. McMillan DC: Systemic inflammation, nutritional status and survival in patients with cancer. Curr Opin Clin Nutr Metab Care 12: 223-226, 2009.

17. Lukaszewicz-Zając M, Mroczko B, Gryko M, Kędra B and Szmitkowski M: Comparison between clinical significance of serum proinflammatory proteins (IL-6and CRP) and classic tumor markers (CEAand CA 19-9) in gastric cancer. Clin Exp Med 11: 89-96, 2011.

18. Shariat SF, Andrews B, Kattan MW, Kim J, Wheeler TM and Slawin KM: Plasma levels of interleukin-6 and its soluble receptor are associated with prostate cancer progression and metastasis. Urology 58: 1008-1015, 2001.

19. Ilhan N, Ilhan N, Ilhan Y, Akbulut H and Kucuksu M: C-reactive protein, procalcitonin, interleukin-6, vascular endothelial growth factor and oxidative metabolites in diagnosis of infection and staging in patients with gastric cancer. World J Gastroenterol 10: 1115-1120, 2004.

20. Lochhead P and El-Omar EM: Gastric cancer. Br Med Bull 85: 87-100, 2008.

21. Hussain SP and Harris CC: Inflammation and cancer: an ancient link with novel potentials. Int J Cancer 121: 2373-2380, 2007. 